\title{
EXPONENTIALS ON LOCALLY COMPACT ABELIAN GROUPS
}

\author{
DAVID NOVAK AND KELLY MCKENNON
}

\begin{abstract}
The canonical mapping on the product of a LCA group with its dual is shown to extend uniquely in a homomorphic and analytic way to the product of the respective complexifications.
\end{abstract}

According to the Pontryagin-Van Kampen theory, locally compact Abelian groups exist in pairs. If $(G, \mathcal{G})$ is one of these pairs, one can either view $G$ as the character group of $\mathcal{G}$, or $\mathcal{G}$ the character group of $G$. For $x \in G$ and $y \in \mathcal{G}$, we shall write the value of $x$ at $y$ (or the value of $y$ at $x$, depending on one's point of view) as $\langle x, y\rangle$.

Let $\Gamma(G)$ be the group of all continuous, nonzero complex-valued, homomorphisms (relative to multiplication in $C$ ).

Then, for each $f$ in $\Gamma(G), 0 \notin f(\mathcal{G})$ and $f$ is the (unique) product of a homomorphism $|f| \mid \mathcal{G} \rightarrow R^{+}$and a character $f /|f|$. Thus $G$ may be regarded as a direct summand of the group $\Gamma(G)$, such that the complementary summand $\Pi(G)$ is isomorphic with the group of continuous homomorphisms $h \mid \mathcal{G} \rightarrow R^{+}$.

The canonical map on $G \times \mathcal{G}$ extends in a trivial way to maps (which we denote also by $\langle$,$\rangle )$

$$
\Gamma(G) \times \mathcal{G} \rightarrow C \text { and } G \times \Gamma(\mathcal{G}) \rightarrow C .
$$

When $G$ is $n$-dimensional Euclidean space $E$, then $\mathcal{G}=E$ as well and

$$
\langle x, y\rangle=e^{i x \cdot y} \quad(\forall x, y \in E)
$$

is the canonical mapping. Here $\Gamma(G)$ may be identified with $n$-dimensional unitary space $U$ (in which $E$ is viewed as a real form) and the extension of the canonical map is

$$
\langle z, y\rangle=e^{i z \cdot y} \quad(\forall z \in U, y \in E) .
$$

In this particular case, however, the extension proceeds even further to a map on $\Gamma(G) \times \Gamma(\mathcal{G})$,

$$
U \times U \ni(a, b) \rightarrow e^{i a \cdot b}
$$

(where $\cdot$ is the inner produce on $U$ ). It is the business of this paper to show that an analogous extension obtains for the general LCA group $G$.

The principal tool employed in studying the extension is a space $\mathfrak{Y}(G)$ of test functions on $G$. This space was introduced in [4] for the purpose of finding as large

Received by the editors December 9, 1979 and, in revised form, May 9, 1980. 1980 Mathematics Subject Classification. Primary 43A40; Secondary 43A25. 
a space of distributions as possible on which the Fourier transform was an isomorphism. It is of intrinsic interest itself and will be the subject of a subsequent paper.

If $a \in \Gamma(G)$ and $b \in \Gamma(\mathcal{S})$, we shall write $\underline{a}$ and $\underline{b}$ for the homomorphisms

$$
\underline{a}|\mathcal{G} \ni y \rightarrow\langle a, y\rangle, \quad \underline{b}| G \ni x \rightarrow\langle x, b\rangle ;
$$

we write $|a|$ and $|b|$ for the elements in $\Pi(G)$ and $\Pi(\mathcal{G})$, respectively, such that

$$
|\underline{a}||\mathcal{G} \ni y \rightarrow| \underline{a}(y) \mid \text { and }|\underline{b}||G \ni x \rightarrow| \underline{b}(x) \mid \text {. }
$$

By convention, we use additive notation for the operations on the Abelian groups $G$ and $\mathcal{G}$.

1. The space of test functions. Let $\mathfrak{A}(G)$ be the set of all $f \in \mathfrak{L}_{1}(G)$ such that $\hat{f} \in \mathfrak{L}_{1}(\mathcal{G})$. As is well known, $\mathfrak{X}(G)$ is a dense ideal of the Banach (convolution) algebra $\mathfrak{L}_{1}(G)$. By $\mathfrak{I}(G)$, we shall mean the set of all functions $f \mid G \rightarrow C$ such that

(i) $\underline{b} f \in \mathfrak{A}(G)$ for all $b \in \Gamma(\mathcal{S})$ and

(ii) $\underline{a}(\underline{b} f)^{\wedge} \in \mathfrak{A}(\mathcal{G})$ for all $(a, b) \in \Gamma(G) \times \Gamma(\mathcal{G})$ (where ^ denotes the Fourier transformation).

For complementary subgroups $S_{1}, S_{2}$ of $G$ and functions $f_{1} \mid S_{1} \rightarrow C$ and $f_{2} \mid S_{2} \rightarrow$ $C$, we define $f_{1} \otimes f_{2} \mid G \rightarrow C$ by

$$
f_{1} \otimes f_{2}\left(s_{1}+s_{2}\right)=f_{1}\left(s_{1}\right) \cdot f_{2}\left(s_{2}\right) \quad\left(\forall s_{1}+s_{2} \in S_{1} \oplus S_{2}=G\right) .
$$

LEMMA 1. Let $G$ be a direct sum $S_{1} \oplus S_{2}$ of closed subgroups and suppose $f_{j} \in \mathfrak{T}\left(S_{j}\right)(j=1,2)$. Then we have $f_{1} \otimes f_{2} \in \mathfrak{T}(G)$.

Proof. We have $\mathcal{G}=\delta_{1} \oplus \delta_{2}$ where $\delta_{1}$ and $\delta_{2}$ are the annihilators of $S_{2}$ and $S_{1}$, respectively, [2, 23.18]. Furthermore, $\delta_{1}$ and $\delta_{2}$ are the duals of $S_{1}$ and $S_{2}$, respectively. Recall that the Haar measure of a product is the product of the Haar measures $[2,15.29]$. Thus

$$
\left(f_{1} \otimes f_{2}\right)^{\wedge}=\hat{f}_{1} \otimes \hat{f}_{2} .
$$

If $a \in \Gamma(G)$ and $b \in \Gamma(\mathcal{S})$ are arbitrary, then $\underline{a}=\underline{a}_{1} \otimes \underline{a}_{2}$ and $\underline{b}=\underline{b}_{1} \otimes \underline{b}_{2}$ for $a_{j} \in \Gamma\left(S_{j}\right)$ and $b_{j} \in \Gamma\left(\delta_{j}\right), j=1,2$. We have

$$
\underline{b}\left(f_{1} \otimes f_{2}\right)=\underline{b}_{1} f_{1} \otimes \underline{b}_{2} f_{2}, \quad \underline{a}\left(\underline{b}\left(f_{1} \otimes f_{2}\right)\right)^{\wedge}=\underline{a}_{1}\left(b_{1} f_{1}\right)^{\wedge} \otimes \underline{a}_{2}\left(\underline{b}_{2} f_{2}\right)^{\wedge},
$$

and

$$
\left(\underline{a}\left(\underline{b}\left(f_{1} \otimes f_{2}\right)\right)^{\wedge}\right)^{\wedge}=\left(\underline{a}_{1}\left(\underline{b}_{1} f_{1}\right)^{\wedge}\right)^{\wedge} \otimes\left(\underline{a}_{2}\left(\underline{b}_{2} f_{2}\right)^{\wedge}\right)^{\wedge} .
$$

That $f_{1} \otimes f_{2}$ is in $\mathfrak{I}(G)$ is now evident. Q.E.D.

For $d \in G$ and $f \mid G \rightarrow C$, we define ${ }_{d} f \mid G \rightarrow C$ by ${ }_{d} f(x)=f(x-d)(\forall x \in G)$.

THEOREM 1. There exists a minimal approximate identity for $\mathfrak{L}_{1}(G)$ in $\mathfrak{I}(G)$.

Proof. The structure theory $[2,24.30]$ provides a decomposition $G=E \oplus H$ in which $E$ may be taken as $n$-dimensional Euclidean space and $H$ a group with compact open subgroup $K$. Consequently $\mathcal{G}=\mathcal{E} \oplus \mathscr{H}$ where

$$
\mathcal{E} \equiv\{y \in \mathcal{G}:\langle x, y\rangle=1(\forall x \in H)\}
$$


and

$$
\mathscr{H} \equiv\{y \in \mathcal{G}:\langle x, y\rangle=1(\forall x \in E)\}
$$

Furthermore [2, 23.18], $\mathcal{E}$ and $\mathcal{H}$ are the duals of $E$ and $H$, respectively-thus $\mathcal{E}$ may also be taken as $n$-dimensional Euclidean space, and the annihilator $\mathcal{F} \equiv$ $\{y \in \mathcal{H}:\langle x, y\rangle=1(\forall x \in K)\}$ of $K$ in $\mathcal{H}$ is a compact, open subgroup of $\mathcal{H}$ $[2,23.29]$. The duality theory $[2,24.11]$ implies that $\mathcal{H} / \mathcal{G}$ is topologically isomorphic to the dual of $K$.

The Banach algebra $\mathfrak{L}_{1}(K)$ admits a minimal approximate identity $\left\{h_{\alpha}\right\}$ consisting of nonnegative, positive-definite functions in $\mathfrak{X}(K)$ such that

$$
\text { the support of each } \hat{h}_{\alpha} \text { is a finite set }
$$

([3, 33.12] and [2, 23.17]). For each index $\alpha$, let $g_{\alpha} \mid H \rightarrow R$ be equal to $h_{\alpha}$ on $K$ and vanish on every other coset of $K$ in $H$. Let $a \in \Gamma(H)$ and $b \in \Gamma(\mathcal{H})$ be arbitrary, and let $\alpha$ be an arbitrary index for the net $\left\{g_{\alpha}\right\}$. The support of $b g_{\alpha}$ being a subset of the compact set $K$, evidently

$$
b g_{\alpha} \in \mathfrak{L}_{1}(H) .
$$

Since $K$ is compact, $\left.\underline{b}\right|_{K}$ is a character and therefore the Extension Theorem $[2,24.12]$ provides an element $d$ of $\mathcal{H}$ such that $\left.\underline{d}\right|_{K}=\left.\underline{b}\right|_{K}$. For all $y \in \mathcal{H}$ we have

$$
\begin{aligned}
\left(\underline{b}_{\alpha}\right)^{\wedge}(y) & =\int_{H} \underline{b}(x) g_{\alpha}(x) \underline{y(x)} d x \\
& =\int_{H} \underline{d}(x) g_{\alpha}(x) \overline{y(x)} d x=\hat{g}_{\alpha}(y-d) .
\end{aligned}
$$

From (1) follows that there exists a finite subset $\mathscr{F}$ of $\mathcal{H}$ such that the cosets $y+\mathcal{F}, y \in \mathscr{F}$, are pairwise disjoint and the support $\operatorname{supp}\left(g_{\alpha}\right)^{\wedge}$ of $\hat{g}_{\alpha}$ is a subset of $\mathcal{F}+\mathcal{G}$. Thus (3) implies that

$$
\operatorname{supp}\left(\left(\underline{b}_{\boldsymbol{\alpha}}\right)^{\wedge}\right) \subset d+\mathscr{F}+\mathcal{g} \text {. }
$$

Since $\mathscr{g}$ is compact, so is $d+\mathscr{F}+\mathcal{F}$ and $\left(\underline{b}_{\alpha}\right)^{\wedge}$ is in $\mathfrak{L}_{1}(\mathcal{F})$. This, with (2), yields

$$
\underline{b} g_{\alpha} \in \mathfrak{X}(H) \text {. }
$$

The compactness of the support of $\left(\underline{b} g_{\alpha}\right)^{\wedge}$ also implies that

$$
\underline{a}\left(\underline{b} g_{\alpha}\right)^{\wedge} \in \mathfrak{R}_{1}(\mathcal{H C}) \text {. }
$$

Since $\left.\underline{a}\right|_{g}$ is a character on $\mathscr{g}$, the Extension Theorem provides an element $\boldsymbol{r}$ of $\boldsymbol{H}$ such that $\left.\underline{r}\right|_{g}=\left.a\right|_{g}$. From (3) we have (where $\xi_{X}$ denotes the characteristic function of $X$ for any $X \subset \mathcal{G}$ )

$$
\left(\underline{b} g_{\alpha}\right)^{\wedge}{ }_{d}\left(\hat{g}_{\alpha}\right)={ }_{d}\left(\sum_{y \in \mathscr{F}} \hat{g}_{\alpha} \xi_{y+g}\right)={ }_{d}\left(\hat{g}_{\alpha}\right) \cdot \sum_{y \in \mathscr{F}}(d+y)\left(\xi_{g}\right)
$$

which yields

$$
\underline{a}\left(\underline{b} g_{\alpha}\right)^{\wedge}{ }_{d}\left(\hat{g}_{\alpha}\right) \sum_{y \in \mathscr{F}} \frac{\underline{a}(d+y)}{r(d+y)} \underline{r}_{(d+y)}\left(\xi_{g}\right) .
$$

Since $\xi_{g}$ is continuous and positive-definite [3, 32.43], it follows from (6) and $[3,32.10]$ that $\underline{a}\left(\underline{b} g_{\alpha}\right)^{\wedge}$ is in the Fourier-Stieltjes algebra $B(G)$ of $G$. Thus, by a 
corollary of Bochner's Theorem [3,33.10], $\left(\underline{a}\left(\underline{b} g_{\alpha}\right)^{\wedge}\right)^{\wedge}$ is in $\mathfrak{L}_{1}(H)$. This, with (5), implies that $\underline{a}\left(\underline{b} g_{\alpha}\right)^{\wedge}$ is in $\mathfrak{A}(\mathcal{H C})$. Hence, in view of (4),

$$
g_{\alpha} \in \mathfrak{I}(H) \text {. }
$$

It is shown in [4, Lemma 4$]$ that there exists a minimal approximate identity $\left\{\nu_{\beta}\right\}$ for $\mathfrak{L}_{1}(E)$ in $\mathfrak{T}(E)$. Thus, by Lemma $1,\left\{\nu_{\beta} \otimes g_{\alpha}\right\}$ is a minimal approximate identity for $\mathfrak{L}_{1}(G)$ in $\mathfrak{I}(G)$. Q.E.D.

LEMMA 2. Let $\mu$ be a complex regular Borel measure on $G$ such that $\int_{G} \underline{b} d|\mu|<$ $\infty$ for all $b \in \Pi(\mathcal{G})$, and let $f$ be in $\mathfrak{I}(G)$. Then $\mu * f$ is in $\mathfrak{T}(G)$ as well.

Proof. Let $a \in \Gamma(G)$ and $b \in \Gamma(\mathcal{G})$ be arbitrary. A direct calculation shows that $\underline{b}(\mu * f)=\underline{b} \mu * \underline{b} f$ (where $\underline{b} \mu$ is the measure whose value at any Borel set $B$ is $\left.\int_{B} \underline{b} d \mu\right)$. Since $\underline{b} \mu$ is by hypothesis a bounded measure, and since $\underline{b} f$ is in $\mathfrak{L}_{1}(G)$ ( $f$ being in $\mathfrak{I}(G)$ ), we have

$$
\underline{b}(\mu * f)=\underline{b} \mu * \underline{b} f \in \mathfrak{L}_{1}(G) .
$$

Furthermore,

$$
\begin{aligned}
\left\|\underline{a}(\underline{b}(\mu * f))^{\wedge}\right\|_{1} & =\left\|\underline{a}(\underline{b} \mu)^{\wedge}(\underline{b} f)^{\wedge}\right\|_{1}<\left\|(\underline{b} \mu)^{\wedge}\right\|_{\infty}\left\|\underline{a}(\underline{b} f)^{\wedge}\right\|_{1} \\
& \leqslant\|\underline{b} \mu\|_{1}\left\|\underline{a}(\underline{b} f)^{\wedge}\right\|_{1}<\infty .
\end{aligned}
$$

When $\underline{a}=1$, (9) means that $\underline{b}(\mu * f)^{\wedge}$ is in $\mathfrak{R}_{1}(\mathcal{S})$-thus, by (8) we have

$$
\underline{b}(\mu * f) \in \mathfrak{A}(G) \text {. }
$$

The equality

$$
\left(\underline{a}(\underline{b}(\mu * f))^{\wedge}\right)^{\llcorner}=\left((\underline{b} \mu)^{\wedge} \underline{a}(\underline{b} f)^{\wedge}\right)^{\llcorner}=(\underline{b} \mu) *\left(\underline{a}(\underline{b} f)^{\wedge}\right)^{\llcorner},
$$

together with the fact that $f$ is in $\mathfrak{T}(G)$, implies that $\left(\underline{a}(\underline{b}(\mu * f))^{\wedge}\right)^{-}$is in $\mathfrak{L}_{1}(G)-$ hence $\underline{a}(\underline{b}(\mu * f))^{\wedge}$ is in $\mathfrak{X}(\mathcal{G})$. Thus, by (10), $\mu * f$ is in $\mathfrak{T}(G)$. Q.E.D.

THEOREM 2. The set $\mathfrak{T}(G)$ is a dense subalgebra of $\mathfrak{L}_{1}(G)$.

Proof. That $\mathfrak{T}(G)$ is an algebra follows from Lemma 2. If $f \in \mathfrak{L}_{1}(G)$ has compact support and if $\left\{f_{\alpha}\right\}$ is the approximate identity of Theorem 1 , then $f * f_{\alpha} \rightarrow f$ in $\mathfrak{L}_{1}(G)$ and Lemma 2 implies that each $f * f_{\alpha}$ is in $\mathfrak{T}(G)$. Thus $\mathfrak{Y}(G)$ is dense in $\mathfrak{L}_{1}(G)$. Q.E.D.

2. The canonical mapping on $\Gamma(G) \times \Gamma(\mathcal{G})$.

Lemma 3. Let $f_{j} \in \mathfrak{T}(G)$ and $x_{j} \in G$ be arbitrary $(j=1,2)$. Then for all $a \in$ $\Gamma(G)$ and $b \in \Gamma(\mathcal{S})$,

(i)

$$
\underline{b}\left(x_{1}\right) \cdot\left(\left(\underline{b} f_{1}\right)^{\wedge} \underline{a}\right)^{\wedge}\left(x_{1}\right) \cdot\left(\hat{f}_{2} \underline{a}\right)^{\wedge}\left(x_{2}\right)=\underline{b}\left(x_{2}\right) \cdot\left(\left(\underline{b} f_{2}\right)^{\wedge} \underline{a}\right)^{\wedge}\left(x_{2}\right) \cdot\left(\hat{f}_{1} \underline{a}\right)^{\wedge}\left(x_{1}\right) .
$$

Proof. We shall have recourse to the easily-verified equalities

$$
\begin{gathered}
\underline{d}(x) \cdot(f \underline{d})^{\wedge} \cdot(-\underline{x})=\left({ }_{x} f \underline{d}\right)^{\wedge} ; \\
(f \underline{d})^{\wedge} * \hat{h}=\hat{f} *(\underline{d h})^{\wedge} ;
\end{gathered}
$$


for all $d \in \Gamma(\mathcal{G}), x \in G$, and $f, h \in \mathfrak{T}(G)$. The left side of (i) equals

$$
\begin{aligned}
& \underline{b}\left(x_{1}\right) \int_{\mathcal{G}} \int_{\mathcal{G}}\left(\underline{b} f_{1}\right)^{\wedge}(y) \underline{a}(y) \underline{x}_{1}(-y) \hat{\hat{f}_{2}}(r) \underline{a}(r) \underline{x}_{2}(-r) d r d y \\
& \quad=\int_{\mathcal{G}} \int_{\mathcal{G}} \underline{b}\left(x_{1}\right) \cdot\left(\underline{b}_{1}\right)^{\wedge}(y) \cdot \underline{x}_{1}(-y) \cdot \hat{f}_{2}(r-y) \cdot-\underline{x}_{2}(r-y) \cdot \underline{a}(r) d r d y
\end{aligned}
$$

(by substituting $r-y$ for $r$ )

$$
\begin{aligned}
& =\int_{\mathcal{G}} \int_{\mathcal{G}}\left({ }_{x_{1}} f_{1} \underline{b}\right)^{\wedge}(y) \cdot\left({ }_{x_{2}} f_{2}\right)^{\wedge}(r-y) \cdot \underline{a}(r) d r d y \\
& =\int_{\mathcal{G}}\left({ }_{x_{1}} f_{1} \underline{b}\right)^{\wedge} *\left({ }_{x_{2}} f_{2}\right)^{\wedge}(r) \cdot \underline{a}(r) d r \\
& =\int_{\mathcal{G}}\left({ }_{x_{1}} f_{1}\right)^{\wedge} *\left({ }_{x_{2}} f_{2} \underline{b}\right)^{\wedge}(r) \cdot \underline{a}(r) d r \quad \text { (by (12)) }
\end{aligned}
$$

which, by symmetry, equals the right side of (i). Q.E.D.

LEMMA 4. Let $(a, b) \in \Pi(G) \times \Gamma(\mathcal{G})$ be arbitrary and let $f \in \mathfrak{T}(G)$ be nonzero. Then the function $C \ni z \rightarrow \int_{\mathcal{G}}(\underline{b} f)^{\wedge}(y) \cdot(\underline{a}(y))^{z}$ dy is entire analytic.

Proof. Evidently $\mathcal{G} \ni y \rightarrow(\underline{a}(y))^{z}$ is a continuous homomorphism of $\mathcal{G}$. Let $\gamma$ be a Jordan curve in the plane and write $m$ and $M$ for elements of $\gamma([0,1])$ whose real parts have minimal and maximal modulus, respectively. Since $f$ is in $\mathfrak{I}(G)$, we have for all $z \in \gamma([0,1])$

$$
\int_{\mathcal{G}}\left|(\underline{b} f)^{\wedge}(y)\right| \cdot\left|\underline{a}(y)^{z}\right| d y<\left\|(\underline{b} f)^{\wedge} \cdot(\underline{a})^{m}\right\|_{1}+\left\|(\underline{b} f)^{\wedge} \cdot(\underline{a})^{M}\right\|_{1}<\infty .
$$

Thus we may apply Fubini's Theorem to obtain

$$
\int_{\gamma} \int_{\mathcal{G}}(\underline{b} f)^{\wedge}(y) \cdot(\underline{a}(y))^{z} d y d z=\int_{\mathcal{G}}(\underline{b} f)^{\wedge}(y) \int_{\gamma}(\underline{a}(y))^{z} d z d y=0
$$

by Cauchy's Theorem. Since $\gamma$ was chosen arbitrarily, Morera's Theorem implies that the function sending each $z \in C$ to

$$
\int_{\mathcal{G}}(\underline{b} f)^{\wedge}(y) \cdot(\underline{a}(y))^{z} d y \text { is analytic. Q.E.D. }
$$

LEMMA 5. For each $a \in \Gamma(G)$, there exists $f \in \mathfrak{T}(G)$ such that $\int_{\mathcal{G}} \hat{f}(y) \underline{a}(y) d y \neq 0$.

Proof. Theorem 2 guarantees the existence of $h \in \mathfrak{T}(G)$ such that $\int_{\mathcal{G}} \hat{h}(y) d y \neq$ 0. Lemma 4 implies that $\left.F\left|C \ni z \rightarrow \int_{\mathcal{G}} \hat{h}(y)\right| \underline{a}(y)\right|^{2} d y$ is entire analytic. Since $0 \neq F(0)$, there exists $r \in R$ such that $0 \neq F(1+i r)$. Let $x \in G$ satisfy $-x=|\underline{a}|^{i r}$. $|a| / \underline{a}$ and define $f$ to be ${ }_{x} h$. Then

$$
0 \neq F(1+i r)=\int_{\mathcal{G}} \hat{h}(y) \underline{x}(-y) \underline{a}(y) d y=\int_{\mathcal{G}} \hat{f}(y) a(y) d y \text {. Q.E.D. }
$$

Lemma 3 can be interpreted as stating that, for each $(a, b) \in \Gamma(G) \times \Gamma(\mathcal{G})$, the number

$$
\frac{\left((\underline{b} f)^{\wedge} \underline{a}\right)^{\wedge}(x)}{(\hat{f} \underline{a})^{\wedge}(x)} \underline{b}(x)
$$


does not depend on the $f \in \mathfrak{I}(G)$ and $x \in G$ employed in its computation (provided, of course, the denominator is nonzero). When $x=0$, then (13) becomes

$$
\frac{\int_{\mathcal{G}}(\underline{b} f)^{\wedge}(y) \cdot \underline{a}(y) d y}{\int_{\mathcal{G}} \hat{f}(y) \cdot \underline{a}(y) d y}
$$

and Lemma 5 guarantees that the denominator can be made nonzero. When $a$ is in $G$, (14) becomes

$$
\frac{(\underline{b} f)^{\wedge \sim}(a)}{\hat{f}^{\sim}(a)}=\underline{b}(a) .
$$

When $b$ is in $\mathcal{G}$,

$$
\int_{\mathcal{G}}(\underline{b} f)^{\wedge}(y) \cdot \underline{a}(y) d y=\int_{\mathcal{G}} \hat{f}(y-b) \cdot \underline{a}(y) d y=\underline{a}(b) \int_{\mathcal{G}} \hat{f}(y) \cdot \underline{a}(y) d y
$$

so (14) becomes $\underline{b}(a)$. Thus, there is no impropriety in defining $\underline{b}(a)$ to be the number in (13) or (14) for any $a \in \Gamma(G)$ and $b \in \Gamma(\mathcal{S})$, and so we do so.

TheOREM 3. For all $a, c \in \Gamma(G)$ and $b, d \in \Gamma(\mathcal{S})$, we have

$$
\underline{b}(a+c)=\underline{b}(a) \cdot \underline{b}(c) \text { and } \underline{b+d}(a)=\underline{b}(a) \cdot \underline{b}(d) \text {. }
$$

Proof. For $x \in G$, we have by (14) and (13)

$$
\begin{aligned}
\underline{b}(a+x) & =\frac{\int_{\mathcal{G}}(\underline{b} f)^{\wedge}(y) \cdot \underline{a+x}(y) d y}{\int_{\mathcal{G}} \hat{f}(y) \cdot \underline{a+x}(y) d y} \\
& =\frac{\left((\underline{b} f)^{\wedge} \underline{a}\right)^{\wedge}(-x)}{(\hat{\hat{f}} \underline{a})^{\wedge}(-x)}=\underline{b}(a) \underline{b}(x) .
\end{aligned}
$$

Thus, the function

$$
F \mid C \ni z \rightarrow \underline{b}(a+z|c|)
$$

equals $\underline{b}(a) \cdot \underline{b}(z|c|)$ for all imaginary $z$ (where $z|c|$ is defined by $\underline{z}|c| \equiv|c|^{z}$ ). In view of Lemma 4 , we thus have

$$
\underline{b}(a+z|c|)=\underline{b}(a) \cdot \underline{b}(z|c|)
$$

for all $z \in C$. Since $c-|c|$ is in $G$, we have

$$
\begin{aligned}
\underline{b}(a+c) & =\underline{b}(a+(c-|c|)+|c|)=\underline{b}(a+|c|) \underline{b}(c-|c|) \\
& =\underline{b}(a) \underline{b}(|c|) \underline{b}(c-|c|)=\underline{b}(a) \underline{b}(|c|+(c-|c|))=\underline{b}(a) \underline{b}(c) .
\end{aligned}
$$

Furthermore, by (14),

$$
\begin{aligned}
\underline{b+d}(a) & =\frac{\int_{\mathcal{G}}(\underline{b} \underline{d} f)^{\wedge}(y) \cdot \underline{a}(y) d y}{\int_{\mathcal{G}}(\underline{d} f)^{\wedge}(y) \cdot \underline{a}(y) d y} \frac{\int_{\mathcal{G}}(\underline{d} f)^{\wedge}(y) \cdot \underline{a}(y) d y}{\int_{\mathcal{G}} \hat{f}(y) \cdot \underline{a}(y) d y} \\
& =\underline{b}(a) \cdot \underline{d}(a) . \quad \text { Q.E.D. }
\end{aligned}
$$

THEOREM 4. The equality $\mathfrak{I}(G)^{\wedge}=\mathfrak{I}(\mathcal{G})$ holds.

Proof. In view of the Fourier Inversion Theorem, it will suffice to show that $\mathfrak{T}(G)^{\wedge} \subset \mathfrak{T}(\mathcal{G})$.

Let $f \in \mathfrak{T}(G)$ and $(a, b) \in \Gamma(G) \times \Gamma(\mathcal{G})$ be arbitrary. Since $a \hat{f}=a(1 \cdot f)^{\wedge}$ is in $\mathfrak{A}(\mathcal{G})$, to show that $\hat{f}$ is in $\mathfrak{T}(\mathcal{G})$, it will suffice to demonstrate that $(\underline{a} \hat{f})^{\wedge} \underline{b}$ is in $\mathfrak{A}(G)$. 
For each $x \in G,(13)$ yields

$$
(a \hat{f})^{\wedge}(x)=\frac{\left((\underline{-b} f)^{\wedge} \underline{a}\right)^{\wedge}(x)}{\langle a,-b\rangle}-b(x)
$$

so

$$
(\underline{a} \hat{f})^{\wedge} \underline{b}=\frac{1}{\langle a,-b\rangle}\left((\underline{-b} f)^{a} \underline{a}\right)^{\wedge} .
$$

Since $f$ is in $\mathfrak{T}(G),(\underline{-b} f)^{\wedge} \underline{a}$ is in $\mathfrak{U}(\mathcal{G})$, whence follows that $\left((\underline{-b} f)^{\wedge} a\right)^{\wedge}$ is in $\mathscr{U}(G)$. Thus, by (15), $(a \hat{f})^{\wedge} \underline{b}$ is in $\mathfrak{A}(G)$ as well. Q.E.D.

COROLlary. Under either convolution or pointwise multiplication, $\mathfrak{Y}(G)$ is an algebra.

Proof. That $\mathfrak{I}(\mathcal{G})$ is an algebra under convolution is a consequence of Theorem 2. Since the Fourier transformation converts convolution to pointwise multiplication, Theorem 4 implies that $\mathfrak{T}(G)$ is closed under pointwise multiplication. Q.E.D.

For $(a, b) \in \Gamma(G) \times \Gamma(\mathcal{G})$ we define $\underline{a}(b)$ by interchanging the roles of $G$ and $\mathcal{G}$.

$$
\underline{a}(b)=\frac{\left((a \underline{f})^{\wedge} \underline{b}\right)^{\wedge}(y)}{(\hat{f} \underline{b})^{\wedge}(y)} \underline{a}(y)
$$

for all $y \in \mathcal{G}$ and $f \in \mathfrak{T}(\mathcal{G})$ for which the denominator is nonzero.

TheOREM 5. For all $(a, b) \in \Gamma(G) \times \Gamma(\mathcal{G})$, we have $\underline{b}(a)=\underline{a}(b)$.

Proof. In view of Theorem 3 , it will suffice to show that $-a(b) \cdot \underline{b}(a)=1$. For $f \in \mathfrak{T}(G)$, we have $\check{f} \in \mathfrak{T}(\mathcal{G})$ and, by (16) and (14),

$$
\begin{aligned}
& \underline{-a}(b) \cdot \underline{b}(a)=\frac{\int_{8}\left(\dot{f} \underline{a}^{-1}\right)^{\wedge}(x) \underline{b}(x) d x}{\int_{8} \check{f}^{\wedge}(x) \underline{b}(x) d x} \frac{\int_{8}(f \underline{b})^{\wedge}(y) \cdot \underline{a}(y) d y}{\int_{8} \hat{f}(y) \cdot \underline{a}(y) d y} \\
& =\frac{\iiint \int \tilde{f}(s) \cdot \underline{a}^{-1}(s) \cdot\langle x,-s\rangle \cdot \underline{b}(x) \cdot f(r) \cdot \underline{b}(r) \cdot\langle r,-y\rangle \cdot \underline{a}(y) d r d s d x d y}{\iiint \int \tilde{f}(s) \cdot\langle x,-s\rangle \cdot \underline{b}(x) \cdot f(r) \cdot\langle r,-y\rangle \cdot \underline{a}(y) d r d s d x d y}
\end{aligned}
$$

$=\frac{\iiint \int \hat{f}(s) \cdot \underline{a}(s) \cdot\langle-r, s\rangle \cdot\langle x, s\rangle \cdot \underline{b}(x) \cdot \underline{b}(-r) \cdot f(r) \cdot \underline{b}(r) \cdot\langle r,-y\rangle \cdot\langle r, s\rangle \cdot \underline{a}(y) \cdot \underline{a}(-s) d r d s d x d y}{\iiint \int \hat{f}(s) \cdot\langle x, s\rangle \cdot \underline{b}(x) \cdot f(r) \cdot\langle r,-y\rangle \cdot \underline{a}(y) d r d s d x d y}$

$=1$.

(substituting $-s$ for $s, x-r$ for $x$, and $y-s$ for $y$ in the numerator successively, and $-s$ for $s$ in the denominator). Q.E.D.

We say that a function $f \mid \Gamma(G) \rightarrow C$ is analytic provided the function mapping each $z \in C$ to $f(z a)$ is analytic for each $a \in \Pi(G)$, where $z a$ is defined by

$$
\underline{z a}(y) \equiv \underline{a}(y)^{z} \quad \text { for all } y \in \mathcal{G} \text {. }
$$

THEOREM 6. The mapping

$$
\psi \mid \Gamma(G) \times \Gamma(\mathcal{G}) \ni(a, b) \rightarrow \underline{a}(b)=\underline{b}(a)
$$

is the only extension of the canonical mapping $\langle$,$\rangle on G \times \mathcal{G}$ to $\Gamma(G) \times \Gamma(\mathcal{S})$ which is homomorphic and analytic in each variable. 
Proof. That $\Gamma(G) \times \Gamma(\mathcal{G}) \ni(a, b) \rightarrow \underline{b}(a)$ is homomorphic in each variable is just Theorem 3. That it is analytic in the first variable follows from (14) and Lemma 4. That it is analytic in the second variable follows from the symmetry implied by Theorem 5 .

Let $\phi$ be an extension to $\Gamma(G) \times \Gamma(\mathcal{G})$ of the canonical map on $G \times \mathcal{G}$ which is homomorphic and analytic in each variable. That $\phi$ is homomorphic in each variable implies that it is determined by its restriction to $\Pi(G) \times \Pi(\mathcal{G})$. Let $(a, b) \in \Pi(G) \times \Pi(\mathcal{G})$ be arbitrary. For each $r \in R$, the map sending each $z \in C$ to $\phi(z a, i r b)$ agrees on the imaginary axis with that sending each $z \in C$ to $\psi(z a, i r b)$. By the Uniqueness Theorem, it follows that

$$
\phi(a, i r b)=\psi(a, i r b) \text { for all } r \in R \text {. }
$$

Since the maps sending each $z \in C$ to $\phi(a, z b)$ and $\psi(a, z b)$ agree on the imaginary axis, it follows that they agree everywhere. In particular, $\phi(a, b)=\psi(a, b)$. Q.E.D.

\section{BIBLIOGRAPHY}

1. P. Eymard, L'algèbre de Fourier d'un groupe localement compact, Bull. Soc. Math. France 92 (1964), 181-236.

2. E. Hewitt and K. Ross, Abstract harmonic analysis, Vol. I, Springer-Verlag, Berlin, 1963.

3. __ Abstract harmonic analysis, Vol. II, Springer-Verlag, Berlin, 1970.

4. K. McKennon, Analytic distributions, J. Reine Angew. Math. 281 (1976), 164-178.

Department of Mathematics, Smmons College, Boston, Massachusetts 02115

Department of Mathematics, Washington State Universtry, Pullman, Washington 99164 